\title{
Zur Frage nach der Muttersubstanz, durch welche die Reaktion von Cammidge im Harn hervorgerufen wird.
}

\author{
Von \\ K. Smolenski, Dozent in St. Petersburg.
}

(Aus dem Nahrungsmittel-Laboratorium des technologischen Instituts zu St. Petersburg.) (Der Redaktion zugegangen am 17. April 1909.)

Seit dem Bekanntwerden der Reaktion von Cammidge, ${ }^{1}$ ) mittels deren es nach Ansicht dieses Forschers in manchen Fällen möglich wird, akute Erkrankungen des Pankreas festzustellen, sind von anderen Forschern mehrere weitere Arbeiten erschienen, die sich die Erforschung des Zusammenhanges zwischen dieser Reaktion und manchen Krankheiten zur Aufgabe stellten. Eine Untersuchung jedoch über die chemische Natur derjenigen Bestandteile des Harns, durch welche diese Reaktion hervorgerufen wird, liegt bis jetzt noch nicht vor.

Wie bekannt, besteht die Reaktion von Cammidge im wesentlichen darin, daß manche Harne, die nicht unmittelbar mit Phenylhydrazin reagieren - also keine Monosaccharide (eventuell Disaccharide) enthalten - diese Reaktion zeigen, sobald sie vorher mit Säuren gekocht worden sind. Augenscheinlich enthalten solche Harne eine kompliziert zusammengesetzte Verbindung, die beim Kochen mit Säuren gespalten wird, wobei ein Körper entsteht, der mit Phenylhydrazin den charakteristischen Niederschlag liefert. Derartige komplizierte Verbindungen wären beispielsweise: Polysaccharide, Glykoside, gepaarte Glukuronsäuren u. a. m. Cammidge selbst nimmt als eine solche «Muttersubstanz» ein kompliziert gebautes Kohlenhydrat, nämlich ein Pentosan an, ohne jedoch einen wirklichen Beweis dafür zu führen.

1) Cammidge, The chemistry of the Urine in diseases of the pancreas. The Lancet 1904. - Cammidge, An improved method of performing the spancreatic reaction s in the urine. Brit. Med. Journ., 1906. 
Durch vorliegende Arbeit soll der erste Versuch gemacht werden, die chemische Natur derjenigen Bestandteile des Harnes, welche die besagte Reaktion bedingen, näher zu untersuchen.

Herr Dr. Petroff, mit dem ich gemeinschaftlich gearbeitet habe, hat die hierzu nötigen klinischen Versuche angestellt, während der chemische Teil von mir ausgeführt worden ist. Die Ergebnisse der Versuche Dr. Petroffs werden im Journal «Russkij Wratsch» veröffentlicht werden.

Es wurde von mir ein Harn mit stark ausgesprochener Reaktion von Cammidge untersucht. Dieses Material stammte von einem Greise, der, wie später die Obduktion feststellte, an Magenkrebs litt; die Pankreasdrüse war jedoch normal.

Vorläufige Untersuchung des Harns.

Der Harn zeigte saure Reaktion; beim Erwärmen mit einem Tropfen Essigsäure wurde keine Eiweißausscheidung beobachtet. Kochen mit Fehlingscher Lösung sowohl direkt als auch nach vorheriger Entfärbung mit Bleiessig (Überschuß von $\mathrm{Pb}$ mit $\mathrm{Na}_{2} \mathrm{SO}_{4}$ entfernt) ergab reichliche fein verteilte Trübung von grau-grüner Farbe; eine Ausscheidung von $\mathrm{Cu}_{2} \mathrm{O}$ fand nicht statt. Beim Erwärmen von $5 \mathrm{ccm}$ Harn mit $1 \mathrm{ccm}$ HCl $\mathrm{d}=1,12$ färbte sich derselbe rasch rosenrot, darauf kirschenrot, schließlich schwarz, und nach anhaltendem Kochen fiel ein dunkelbrauner Niederschlag aus. Hierbei wurde jedoch keine Bildung von Furfurol beobachtet (Reaktion auf Anilinpapier); dagegen traten Dämpfe von scharfem widerlichem Geruch auf. ${ }^{1}$ )

Der mit etwas $\mathrm{HCl}$ erwärmte und darauf mit $\mathrm{NaOH}$ neu-

1) Es wurde noch der Versuch gemacht, die beim Kochen des Harns mit Säure entstehenden flüchtigen Bestandteile näher zu bestimmen. $100 \mathrm{ccm}$ Harn $+2 \mathrm{ccm}$ konzentrierter $\mathrm{H}_{2} \mathrm{SO}_{4}$ wurden $\mathrm{zu}$ diesem Zwecke auf dem Sandbade abdestilliert (Kochdauer 25 Min.). Es resultierten $20 \mathrm{ccm}$ eines farblosen Destillates von widerlichem Geruch, das, mit Lackmuspapier geprüft, stark saure Reaktion zeigte.

In Anbetracht dessen, daß bei starker Glukosurie stets Acetessigsäure im Harn vorhanden ist, wurde auch das erhaltene Destillat auf Aceton geprüft, doch gelang es weder mit $J$ (Bildung von Jodoform) noch mit o-Nitro-p-Benzaldehyd (Bildung von Indigo) eine Reaktion zu erhalten. 
tralisierte Harn reduzierte kräftig $\mathrm{F}$ ehling sche Lösung beim Erwärmen; es bildeten sich Niederschläge von gelblich bis rötlich brauner Farbe, die jedoch von der gleichzeitig reichlich ausfallenden feinen grau-grünlichen Trübung zum Teil maskiert wurden.

Jedenfalls erlaubt uns das Verhalten gegen Fehling sche Lösung, den Schluß zu ziehen, daß im Harn ein Körper enthalten sein muß, welcher zwar nicht unmittelbar, wohl aber nach dem Kochen mit Säuren Fehlingsche Lösung reduziert.

Mit $\alpha$-Napthol und $\mathrm{H}_{2} \mathrm{SO}_{4}$ zeigte der Harn eine intensive Reaktion; übrigens wurde schon beim Zusatz von $\mathrm{H}_{2} \mathrm{SO}_{4}$ allein eine rasch auftretende dunkle Färbung beobachtet.

Die Untersuchung des mit Bleiessig entfärbten Harns im Polarisationsapparate $^{1}$ ) ergab eine Drehung von $+0,9^{\circ}$ der Saccharimeterskala.

Durch Zusatz eines $4-5$ fachen Volumens absoluten Alkohols zum Harn wurde in reichlicher Menge ein leichter, reiner flockiger Niederschlag ausgeschieden, der sich rasch zu Boden setzte.

Mit Phenylhydrazin erfolgte unmittelbar keine Reaktion. $50 \mathrm{ccm}$ Harn $+10 \mathrm{~g}$ essigsaures $\mathrm{Na}+3,7 \mathrm{~g}$ HCl-Phenylhydrazin $+2 \frac{1}{2} \mathrm{ccm}$ Eisessig wurden 20 Minuten auf dem kochenden Wasserbade erwärmt; es resultierte nur eine schwache Trübung, die sich beim Stehen nicht vermehrte.

Dagegen zeigte der Harn in ausgesprochener Weise die Reaktion von Cammidge: $50 \mathrm{ccm}$ Harn $+5 \mathrm{~cm} \mathrm{HCl}$ von $\mathrm{d}=1,19$ wurden im Sandbade 10 Minuten gekocht (hierbei Dunkelfärbung und Auftreten scharfer Dämpfe beobachtet). Der abgekühlte Harn wurde mit $25 \mathrm{ccm} \mathrm{H}_{2} \mathrm{O}$ verdünnt und mit $20 \mathrm{~g} \mathrm{PbCO}_{3}$ neutralisiert, filtriert, zum Filtrat $10 \mathrm{~g}$ essigsaures $\mathrm{Na}+3,75 \mathrm{~g} \mathrm{HCl}$-Phenylhydrazin $+2^{1 / 2} \mathrm{ccm}$ Eisessig zugesetzt und 10 Minuten auf dem Sandbade schwach gekocht. Beim Abkühlen resultierte in reichlicher Menge ein leichter, gelber flockiger Niederschlag, der seinem Aussehen nach vollkommen dem charakteristischen Glukosazonniederschlage glich. Der abgesaugte, mit $\mathrm{H}_{2} \mathrm{O}$ gewaschene und über $\mathrm{H}_{2} \mathrm{SO}_{4}$ getrocknete

1) Es gelangte ein empfindlicher geradliniger Saccharimeter mit doppelter Keilkompensation der Firma Julius Peters zur Anwendung. 
Niederschlag zeigte einen Schmelzpunkt von $193-194^{\circ}$, erwies sich also identisch mit dem Schmelzpunkt des Galactosazons.

Da die tägliche Nahrung des Patienten in $1 \frac{1}{1} 2$ Glas Kefir bestand, lag die Annahme nahe, daß der im Kefir enthaltene Milchzucker in Dextrose und Galactose gespalten, welch letztere aus Gründen unbekannter Art vom Organismus nicht assimiliert wird, sondern in Verbindung mit einem unbekannten Stoff im Harn zur Ausscheidung gélangt (ähnlich wie die gepaarte Glukuronsäure?).

Die erwähnte Bildung eines reichlichen Niederschlages durch absoluten Alkohol führte mich auf den Gedanken, daß im Harn möglicherweise ein dem tierischen Gummi ähnlicher Körper vorhanden sein könnte, welcher bei der Hydrolyse Galactose liefert (Galactose entsteht fast regelmäßig bei der Hydrolyse von Pflanzengummi und Pflanzenschleim); vielleicht ein Galactan, ähnlich denjenigen, wie sie von verschiedenen Forschern aus Pflanzenstoffen erhalten worden sind. Dieses vermutete Galactan versuchte ich daher aus dem Harn zu isolieren:

1. durch Fällung mit absolutem Alkohol;

2. durch Abscheidung der im Harn enthaltenen Kohlenhydrate in Form ihrer Benzoylester.

Untersuchung des aus dem Harn durch absoluten Alkohol gefällten Niederschlages.

$300 \mathrm{ccm}$ Harn $+800 \mathrm{ccm}$ absoluten Alkohols $+100 \mathrm{ccm}$ Äther. Der reichlich ausgeschiedene Niederschlag wurde abgesaugt, mit Alkohol und Äther ausgewaschen und getrocknet. Derselbe erwies sich als fast ausschließlich aus anorganischen Stoffen, vorwiegend Mg-Salzen bestehend. Organische Verbindungen, speziell Kohlenhydrate, waren kaum vorhanden. Ein derartiges Resultat zog natürlich die Voraussetzung des Vorhandenseins eines gummiartigen Körpers (Galactans) im Harn wiederum stark in Zweifel.

Abscheidung der im Harn enthaltenen Kohlenhydrate in Form ihrer Benzoylester.

Gemäß den in der Literatur vorhandenen Angaben erhielt ich die Benzoylester auf folgende Art: 
Zwecks Ausscheidung der Erdalkaliphosphate wurden $250 \mathrm{ccm}$ Harn mit $5 \mathrm{ccm} 10 \%$ iger $\mathrm{NaOH}$ versetzt; zur abfil-

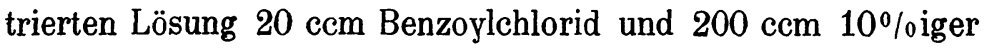
Natronlauge zugefügt und das Gemisch so lange geschüttelt, bis der Geruch nach Benzoylchlorid fast verschwunden war. Hierbei schieden sich die Benzoate teils in Form eines zu Boden fallenden krystallinischen Niederschlages, teils als klebrige auf der Oberfläche des Reaktionsgemisches schwimmende Masse aus. Sowohl der auf die eine wie die andere Art ausgeschiedene Niederschlag zeigte in Alkohol gelöst mit $\alpha$-Naphthol eine äußerst intensive Reaktion auf Kohlenhydrate. Die abgesaugten und mit Wasser gewaschenen Benzoate wurden in $150 \mathrm{ccm}$ einer alkoholischen Lösung von Natriumäthylat $(3 \mathrm{~g}$ metallisches Natrium) gelöst, nach erfolgter Verseifung $150 \mathrm{ccm} \mathrm{H}_{2} \mathrm{O}+7 \mathrm{ccm}$ konzentrierte Schwefelsäure zugefügt und durch dreimaliges Ausschütteln mit Äther im Scheidetrichter die Benzoesäure aus der Lösung entfernt.

Die wässerige Schicht, mit dem Wasser, welches zum Waschen der Ätherschicht diente, vereinigt, zeigte folgende Reaktionen:

1. Mit $\alpha$-Naphthol $+\mathrm{H}_{2} \mathrm{SO}_{4}$ starke Violettfärbung, also Kohlenhydrate vorhanden;

2. Mit Fehlingscher Lösung nach erfolgter Neutralisation mit $\mathrm{NaOH}$ gekocht: keine Trübung. Bildung eines geringen fast rein roten Niederschlages von $\mathrm{Cu}_{2} \mathrm{O}$;

3. Nach vorhergegangenem Erwärmen mit einem Tropfen $\mathrm{H}_{2} \mathrm{SO}_{4}$ während 5 Minuten und Neutralisation mit $\mathrm{NaOH}$ mit Fehlingscher Lösung gekocht, erfolgte reichliche Bildung eines rein roten Niederschlages von $\mathrm{Cu}_{2} \mathrm{O}$.

Somit war es offenbar gelungen, aus dem Harn ein Polysaccharid in Form seines Benzoylesters zu isolieren und aus letzterem dieses Kohlenhydrat in freiem Zustand abzuspalten.

Die die angeführten Reaktionen zeigende wässerige Lösung wurde mit $\mathrm{Ba}(\mathrm{OH})_{2}$ neutralisiert, vom gebildeten $\mathrm{BaSO}_{4}$ abfiltriert und das schwach alkalische Reaktion zeigende Filtrat bis auf $100 \mathrm{ccm}$ eingeengt. 
Die Polarisation dieser Lösung ergab nur $+0,3^{\circ}$, also war es offenbar nur einen geringen Teil der im Harn enthaltenen Kohlenhydrate zu erhalten gelungen. Darauf wurde die Lösung noch weiter eingeengt und mit absolutem Alkohol gefällt. Der reichlich ausgeschiedene Niederschlag von Natriumsulfat enthielt keine organischen Substanzen (Kohlenhydrate), eine Tatsache, durch welche nochmals die Annahme des Vorhandenseins von tierischem Gummi im Harn hinfällig wird. Durch nochmaliges Eindampfen und Fällen mit absolutem Alkohol wurden wiederum nur mineralische Stoffe ausgeschieden. Das auf $100 \mathrm{ccm}$ aufgefüllte Filtrat ergab bei der Polarisation $+0,2^{\circ}$.

Die Lösung wurde hierauf durch Zusatz von 5 Tropfen konzentrierter $\mathrm{H}_{2} \mathrm{SO}_{4}$ und Erwärmen auf dem Wasserbade während 10 Minuten hydrolysiert. Nunmehr ergab die Polarisation $-0,05^{\circ}$, Fehlingsche Lösung wurde stark reduziert. Mit einem Resorcinkrystalle und $\mathrm{HCl}$ erwärmt, entstand rasch eine schöne Rotfärbung; beim Stehen schied sich hierbei ein amorpher, ebenfalls roter Niederschlag aus, der sich in Alkohol mit roter Farbe löste. Diese letzte Reaktion läßt auf das Vorhandensein von Lävulose in der hydrolysierten Lösung schließen.

Berücksichtigt man ferner die Polarisation der Lösung vor und nach der Hydrolyse, sowie das Verhalten gegen Fehlingsche Lösung (und Phenylhydrazin), so kommt man unwillkürlich auf den Gedanken, daß die ursprüngliche Substanz, welche die Reaktion von Cammidge hervorruft, nichts anders als Saccharose sein könnte.

Zur Prüfung der Richtigkeit dieser Annahme wurden folgende Untersuchungen ausgeführt:

1. Prüfung der optischen Eigenschaften des Harns vor und nach erfolgter Hydrolyse (Inversion);

2. Untersuchung des mit Phenylhydrazin nach Cammidge abgeschiedenen Niederschlages;

3. Versuch der Isolierung des Polysaccharides aus dem Harn in Form seiner Strontianverbindung nach der Methode von E. Schulze. 
Die optischen Eigenschaften des Harns vor und nach erfolgter Inversion.

Die direkte Polarisation wurde im Harn, der vorher mit Bleiessig ( $1 / 10$ Volumen) entfärbt war, ausgeführt. Darauf wurde $\mathrm{HCl}$ oder $\mathrm{H}_{2} \mathrm{SO}_{4}$ in geringer Menge zugefügt und auf dem Wasserbade erwärmt. Die sich stark färbende Lösung mit trockenem Bleicarbonat entfärbt, filtriert und das Filtrat polarisiert.

Diese Bestimmungen wurden häufig und zu verschiedenen Zeiten ausgeführt.

Die direkte Polarisation schwankte zwischen $+0,6^{\circ}$ und $0,9^{\circ}$ der Saccharimeterskala; nach der Inversion - von $0^{0}$ bis $-0,2^{\circ}$. In denjenigen Fällen, in denen die Inversion nach der für die Inversion von Saccharose gebräuchlichen Vorschrift (nämlich: $5 \mathrm{ccm} \mathrm{HCl} \mathrm{d}=1,19$ auf $75 \mathrm{ccm}$ Harn, während 5 Minuten auf $68-70^{\circ}$ erwärmt) ausgeführt wurde, stimmte die direkte Polarisation genau mit der nach der Inversion ermittelten überein, unter der Voraussetzung, daß das untersuchte Kohlenhydrat Saccharose ist.

Es wurde gefunden:

1. Direkte Polarisation $\mathrm{P}=+0,75^{\circ}$ der Saccharimeterskala, Inversionspolarisation $\mathrm{J}=-0,20^{\circ}$, bei $\mathrm{t}=20^{\circ} \mathrm{C}$.

Nach der Formel $Z=\frac{P+J}{141,84+\frac{J}{20}-\frac{t}{2}}$ berechnet, $Z=+0,72^{\circ}$. $\left(0,75^{\circ}\right.$ der Saccharimeterskala entspricht $0,2 \mathrm{~g}$ Saccharose in $100 \mathrm{ccm}$ Harn.)

2. $\mathrm{P}=+0,6^{\circ} ; \mathrm{J}=-0,15^{0} ; \mathrm{Z}=+0,57^{\circ}$.

In denjenigen Fällen, in welchen die Inversion bei höherer Temperatur, mit größeren Säurequanta und längerer Dauer der Erwärmung stattfand, zeigte die Inversionspolarisation einen geringeren Gehalt an Saccharose an, offenbar infolge des Zerfalls der Lävulose, die sich ja, wie bekannt, gegen Säuren nur wenig widerstandsfähig erweist.

Untersuchung des durch die Reaktion von Cammidge entstehenden Niederschlages.

Derselbe wurde zweimal dargestellt, wobei größere Harnmengen, nämlich jeweils $300 \mathrm{ccm}$ zur Verarbeitung gelangten. 
Bei der Darstellung wurde mit geringen Abweichungen die Vorschrift von $\mathrm{G}$ ammidge eingehalten.

Der Harn wurde zunächst mit Bleiessig gereinigt. Statt Salzsäure wandte ich zur Hydrolyse Schwefelsäure an, weil diese Säure bei der Neutralisation mittels Bleicarbonat das unlösliche Bleisulfat liefert und daher leicht aus der Lösung entfernt werden kann. Statt des salzsauren Phenylhydrazins wandte ich die freie Base mit der erforderlichen Menge Eisessig an. $300 \mathrm{ccm}$ mittels Bleiessig entfärbten Harns wurden mit $10 \mathrm{ccm}$ Schwefelsäure während 10 Minuten auf dem Sandbade gekocht, die Lösung abgekühlt, Bleicarbonat bis zu erfolgter Neutralisation zugesetzt, abgesaugt und der Niederschlag mit wenig Wasser gewaschen. Zum Filtrat wurden $60 \mathrm{~g} \mathrm{Na}-$ triumacetat $+18 \mathrm{ccm}$ freies Phenylhydrazin $+25 \mathrm{ccm}$ Eisessig zugefügt und 10 Minuten gekocht. Nach dem Erkalten fiel ein flockiger gelber Niederschlag aus, der unter dem Mikroskop sich als aus feinen, spitzen, gelben Nadeln bestehend erwies. Am folgenden Tage wurde der erhaltene Niederschlag abfiltriert, ausgewaschen und über Schwefelsäure getrocknet. Die Menge des erhaltenen Niederschlages betrug in einem Falle 0,6 g. Der Schmelzpunkt dieses rohen Osazons war in einem Falle $189^{\circ}$, in einem anderen $193^{\circ}$.

Zur Reinigung wurde der Niederschlag zwecks Entfernung harziger Beimengungen mit kaltem Äther ausgezogen und darauf aus Alkohol von 50-60\%, in welchem er sich leichter löslich als in absolutem Alkohol erwies, umkrystallisiert. Beim Erkalten der in der Siedehitze gesättigten Lösung fiel ein schöner gelber krystallinischer Niederschlag aus, der unter dem Mikroskop charakteristisch zu garben- oder -pinselartigen Büscheln vereinigte gelbe Nadeln zeigte, wie solche genau im mikroskopischen Bilde des Glukosazonniederschlages beobachtet werden. Der Schmelzpunkt des umkrystallisierten Osazons betrug : $1.202^{\circ}$, 2. $205-206^{\circ}$.

Der durch die Reaktion von Cammidge erhaltene Niederschlag ist also offenbar Glukosazon, das ja auch aus den Hydrolyseprodukten der Saccharose, d. h. aus d-Glukose und d-Fruktose erhalten werden muß. 
Bemerken möchte ich bei dieser Gelegenheit, daß das Glukosazon aus dem Harn, je nach den Reaktionsbedingungen, mehr oder minder kompakt ausfallen kann und daher eine Schätzung der Menge des gebildeten Niederschlages nach Augenmaß zu fehlerhaften Schlüssen führen kann.

Abscheidung von Saccharose aus dem Harn.

Da sämtliche Untersuchungsergebnisse zur Annahme berechtigten, daß der Harn des Patienten Saccharose enthielt die also, ohne im Organismus gespalten noch von demselben assimiliert zu werden (wohl infolge mangels der entsprechenden Enzyme oder ungeeigneter Bedingungen für deren Wirkung), in das Blut gelangt und durch die Nieren mit dem Harn ausgeschieden wird - - wurde am Patienten ein Ernährungsversuch mit Saccharose angestellt. Für gewöhnlich nahm der Patient täglich nur 2 Stück Zucker in Tee gelöst zu sich. Nunmehr wurden ihm von Dr. Petroff täglich $100 \mathrm{~g}$ Zucker, in mehrmaligen Dosen gereicht, ordiniert. Nach vorhandenen Literaturangaben sowie nach Beobachtungen Petroffs läßt sich beim täglichen Konsum dieses Quantums im Harn eines gesunden Menschen noch kein Rohrzucker nachweisen.

Der Harn des Kranken zeigte nun während mehrerer Tage die Cammidgesche Reaktion in stärkster Form.

Die Polarisation dieses Harns ergab:

$\mathrm{P}=+3,2^{\circ}$; entsprechend $0,83 \mathrm{~g}$ Zucker in $100 \mathrm{ccm}$;

$\mathrm{J}=-1,1^{0}$;

$\mathrm{Z}=+3,26^{\circ}$.

Die direkte Polarisation stimmte also mit der nach der Inversionsmethode erhaltenen völlig überein, wie solches bei Saccharose zutrifft.

Der nach Cam mid ge erhaltene Niederschlag aus 60\% igem Alkohol umkrystallisiert zeigte einen Schmelzpunkt von $203^{\circ}$, sowie das charakteristische mikroskopische Bild.

Zwecks Abscheidung der Saccharose aus diesem Harn wurden $300 \mathrm{ccm}$ mit Bleiessig gefällt, filtriert, Schwefelwasserstoff eingeleitet, das gebildete Schwefelblei abfiltriert, mit Natron neutralisiert und bei Einhaltung fast neutraler Reaktion stark 
eingeengt. Hierauf wurde ein 5-6faches Volumen an absolutem Alkohol zugegeben, die entstandene Trübung abfiltriert, das Filtrat zum Kochen erhitzt, mit einer heißen, gesättigten, wässerigen Lösung von $5-6 \mathrm{~g} \mathrm{Sr}(\mathrm{OH})_{2} \cdot 8 \mathrm{H}_{2} \mathrm{O}$ versetzt und 10-15 Minuten gekocht, wobei ein reichlicher Niederschlag entstand. Nach dem Erkalten wurde sofort abgesaugt, der Niederschlag mit Alkohol gewaschen, im Wasser suspendiert und mit Kohlensäure zerlegt. Die von Strontiumcarbonat befreite Lösung wurde eingeengt, mit Alkohol auf $100 \mathrm{ccm}$ aufgefüllt und polarisiert: $\mathrm{P}=+4,1^{\circ}$; schließlich aufs neue eingeengt, mit Alkohol versetzt, die entstandene Trübung abfiltriert, zum Filtrat wiederum absoluter Alkohol bis zum Beginn einer bleibenden Trübung zugefügt, und die Lösung über Ätzkalk der Krystallisation überlassen. Nach einigen Tagen wurden kleine, glänzende, harte Kryställchen erhalten, welche, unter dem Mikroskop betrachtet, die für Saccharose charakteristische Form zeigten. Nach beendigter Krystallisation wurde die gesamte Krystallmasse in wenig Wasser gelöst, zur Klärung mit etwas Tonerdehydrat und Bleiessig versetzt und auf $25 \mathrm{ccm}$ aufgefüllt.

Die Polarisation ergab:

$\mathrm{P}=+13,3^{\circ}$; entsprechend $0,9 \mathrm{~g}$ Saccharose in $25 \mathrm{ccm}$;

$\mathrm{J}=-3,5^{0}$;

$\mathrm{Z}=+12,8^{\circ}$.

Fehlingsche Lösung wurde vor der Inversion nicht, nach derselben jedoch stark reduziert; mit Resorcin wurde die Reaktion auf Lävulose erhalten.

Es unterliegt keinem Zweifel, daß das erhaltene Kohlenhydrat Saccharose ist.

Weiter wurde ein Versuch angestellt, die Saccharose auch aus dem Harn abzuscheiden, welcher aus der Zeit stammte, da der Patient täglich nur 2 Stück Zucker erhielt.

Dieser Harn zeigte eine Polarisation von $0,6-0,8^{\circ}$. In der oben beschriebenen Weise wurden $750 \mathrm{ccm}$ Harn von $\mathrm{P}=+0,6^{\circ}$ verarbeitet und die Saccharose mit $\mathrm{Sr}(\mathrm{OH})_{2}$ zur Abscheidung gebracht.

Wiederum gelang es, Krystalle zu erhalten, allerdings in weit geringerer Menge. In $25 \mathrm{ccm}$ gelöst ergaben dieselben: 
$\mathrm{P}=+5,7^{\circ}$; entsprechend $0,37 \mathrm{~g}$ Saccharose in $25 \mathrm{ccm}$;

$\mathrm{J}=-1,9^{\circ}$;

$\mathrm{Z}=+5,77^{\circ}$.

Die Lösung zeigte dieselben Reaktionen.

Auch in diesem Falle erscheint es somit außer Zweifel, daß das ausgeschiedene Kohlenhydrat Saccharose ist.

Durch die angeführten Versuche halte ich es für völlig erwiesen, daß das Kohlenhydrat, welches in dem von mir untersuchten Harn die Reaktion von Cammidge hervorrief, nur Saccharose sein kann. Zwar läßt sich diese Tatsache noch nicht derart verallgemeinern, um aus derselben den Schluß zu ziehen, daß die Cammidgesche Reaktion stets durch die Anwesenheit von Saccharose bedingt wird; wäre dieses aber der Fall, so könnte das Auftreten derselben darauf hindeuten, daß bei manchen Erkrankungen die Saccharose vom Organismus nicht assimiliert wird, sondern unzersetzt zur Ausscheidung gelangt.

Jedenfalls möchte ich dringend raten, bei Ausführung der Reaktion stets auch eine Polarisation des Harns sowohl direkt als auch nach der Inversionsmethode auszuführen und auch den Schmelzpunkt des erhaltenen Niederschlages $z u$ bestimmen.

Weiter dürfte sich auch stets ein Ernährungsversuch mit Saccharose empfehlen, um eine eventuelle Verstärkung der Reaktion wahrnehmen zu können.

Vielleicht würde es auf diese Weise gelingen, einen $\mathrm{Zu}-$ sammenhang zwischen manchen Erkrankungen und der Anwesenheit von Saccharose im Harn (für welche Erscheinung ich den Namen «Saccharosurie» vorschlage) festzustellen.

Zum Schluß seien noch weitere in Gemeinschaft mit Dr. Petroff angestellte Ernährungsversuche mit anderen Kohlenhydraten sowie die Untersuchungsergebnisse der hierbei erhaltenen Harne angeführt.

Der Patient erhielt zu verschiedenen Zeiten: 1. $25 \mathrm{~g}$ Lactose, 2. $50 \mathrm{~g}$ Dextrin, 3. $25 \mathrm{~g}$ Galactose. In allen drei Fällen wurde keine merkbare Verstärkung der Cammidg eschen Reak- 
130 K. Smolenski, Über die Reaktion von Cammidge im Harn.

tion konstatiert, d. h. es erfolgte keine Vermehrung an Kohlenhydraten im Harn.

Die Polarisation ergab:

bei Dextrinernährung: $\mathrm{P}=+0,7^{\circ}$,

$$
\mathrm{J}=-0,2^{0}
$$

bei Galactoseernährung $\mathrm{P}=+0,4^{\circ}$,

$$
\mathrm{J}=-0,1^{\circ} \text {. }
$$

Schmelzpunkt des Niederschlages:

bei Dextrinernährung $203^{\circ}$ (umkrystallisiert);

bei Galactoseernährung $185-186^{\circ}$ (aus Mangel an Substanz nicht umkrystallisiert).

Aus den erhaltenen Resultaten läßt sich schließen, daß sowohl Lactose als auch Dextrin und Galactose vom Organismus des Patienten völlig assimiliert wurden. Offenbar verlief die Hydrolyse der Lactose und Dextrin völlig normal. Nur die Saccharose konnte nicht gespalten werden und gelangte daher als solche in den Harn. 